Revista de Economia Política, vol. 29, $n^{\circ} 3$ (115), pp. 114-134, julho-setembro/2009

\title{
Diferentes padrões de políticas salariais nos estados brasileiros: uma análise a partir do diferencial de salários público-privado
}

\author{
GABRIELA MIRANDA MORICONI \\ JOÃO S. MOURA NETO \\ NELSON MARCONI \\ PAULO ROBERTO ARVATE*
}

\begin{abstract}
Public servant wages represent a significant part of Brazilian state governments' current expenses. Based on the literature, this paper has tried to identify which states practice efficient, compensatory or appropriation wage politics through the estimation of public-private wage differentials. The differential of salaries was calculated between 1995 and 2004 following the Oaxaca's technique. The results show that the wage policy of an important number of states has had efficiency and a numerous part of the North and the Northeast states have developed compensatory politics. The Federal District and Roraima practiced a wage policy characterized by explicit appropriation.

Keywords: public-private differential; wages; public expenditure.

JEL Classification: H76; J31; J4.5
\end{abstract}

\section{INTRODUÇÃO}

Uma parcela considerável das despesas correntes dos governos estaduais brasileiros é direcionada para o pagamento de salários dos seus funcionários. Segundo dados disponibilizados pelo IPEA (Instituto de Pesquisa Econômica Apli-

\footnotetext{
* Respectivamente, doutoranda em Administração Pública e Governo na Escola de Administração de Empresas de São Paulo da Fundação Getulio Vargas, e-mail: gabi.mariconi@gmail.com; Escola Superior de Administração Marketing e Comunicação, Sorocaba. E-mail:joaomoura@gvmail.br; Fundação Getulio Vargas e Pontifícia Universidade Católica de São Paulo. E-mail: nelson.marconi@fgv.br; Escola de Administração da Fundação Getulio Vargas de São Paulo; Escola de Economia da Fundação Getulio Vargas de São Paulo Centro de Estudos de Política e Economia do Setor Publico. E-mail: paulo.arvate@fgv.br
} 
cada), a média de gastos com salários por governo estadual brasileiro entre os anos de 1985 e 1994 supera o patamar de 70\%. Não existem diferenças de comportamento em relação a essa média, sejam estados de regiões ricas, sejam estados de regiões pobres. Pode-se então inferir que a política salarial dos estados é a mesma?

De acordo com a literatura sobre o tema, tanto o nível de gastos com salários nas despesas correntes quanto o nível salarial isoladamente não seriam bons indicadores da política salarial realizada. Uma resposta adequada na tentativa de compreender os objetivos da política salarial escolhida estaria na comparação da remuneração entre o setor público e o setor privado, por meio do cálculo do diferencial de salários. Borjas (1986), Gyourko e Tracy (1991), Katz e Krueger (1991) e Poterba e Rueben (1998) são exemplos de trabalhos que encontram resultados heterogêneos para os diferenciais de salário entre os trabalhadores do setor público e do setor privado dos governos subnacionais americanos. Estes são resultados que, em nosso entender, indicariam a existência de práticas salariais distintas entre esses governos. Para o caso brasileiro, poucos são os avanços de estudos na área de diferencial de salários público-privado, especialmente em se tratando de governos subnacionais ${ }^{1}$.

Dessa forma, este trabalho possuirá dois objetivos. Primeiro, investigar se o diferencial de salários público-privado é homogêneo ou heterogêneo entre os estados brasileiros. Em segundo lugar, pretende-se estabelecer uma metodologia para que se possa identificar a presença de determinados padrões na política salarial dos estados.

Este trabalho pretende avançar na literatura porque, a despeito de existirem argumentos indicando os objetivos do estabelecimento de uma política salarial no âmbito do setor público, não foi encontrada a construção de uma metodologia que enquadre os diferentes comportamentos dos governos na amostra estudada.

Este trabalho terá mais quatro seções além desta introdução. Na segunda seção apresentaremos a discussão teórica a respeito da determinação dos salários no setor público e uma revisão de estudos empíricos a respeito do diferencial de salário público-privado. Na terceira seção apresentaremos a metodologia de cálculo dos diferenciais de salário entre os setores público e privado e os resultados encontrados para os estados brasileiros entre os anos de 1995 e 2004 . Na quarta seção apresentaremos a metodologia e classificação das políticas salariais nos estados brasileiros de acordo com a taxonomia encontrada na literatura. Na última seção, apresentaremos nossas principais conclusões a respeito do comportamento dos governos estaduais na definição de suas políticas salariais.

\footnotetext{
${ }^{1}$ Os trabalhos de Barros et al. (2000) e Marconi (2004) estão entre as poucas exceções.
} 


\section{A LITERATURA SOBRE A EXISTÊNCIA DE DIFERENCIAIS DE SALÁRIO ENTRE O SETOR PÚBLICO E O SETOR PRIVADO}

\section{A determinação da política salarial no setor público}

A discussão a respeito do estabelecimento dos níveis salariais no setor público tem sido constantemente permeada pela comparação destes com os salários pagos pelo setor privado. Fogel e Lewin (1974) indicam que o início desta prática está relacionado à reivindicação de servidores públicos americanos por salários no mínimo equivalentes àqueles pagos aos trabalhadores de ocupações semelhantes no setor privado de seu país.

A literatura sobre o tema seguiu utilizando o setor privado como parâmetro de comparação, especialmente para avaliar a eficiência do setor público na definição dos seus níveis de salário. Isto se deve ao fato de que o setor privado, ao operar sob a lógica da acumulação, encontraria restrições no processo de determinação dos salários de seus trabalhadores, pois os salários corresponderiam ao preço de apenas um dos fatores de produção - a força de trabalho - cujos custos devem ser minimizados para que se consiga maximizar os lucros. Na ausência das restrições aos salários encontradas no setor privado, Fogel e Lewin (1974) defendem que a opção mais eficiente para o setor público seria o pagamento aos servidores de salários equivalentes aos que seus contrapartes no setor privado recebem, de forma a atrair ao governo profissionais que tenham pelo menos a qualificação média existente no mercado de trabalho privado. De acordo com estes autores, o pagamento de salários mais elevados por parte do governo em relação aos níveis remuneratórios observados no setor privado seria desnecessário e desperdiçaria receitas públicas ${ }^{2}$.

No entanto, existem evidências de que nem sempre estas são as práticas salariais adotadas pelos formuladores de políticas públicas, especialmente em razão das distinções entre os ambos os setores. Enquanto o setor privado atua com o objetivo de maximização de lucros e pelo princípio da troca, o setor público possui objetivos distintos, como por exemplo a equidade social, bem como baseia sua atuação no princípio da transferência, no sentido de que o Estado arrecada recursos de determinados grupos da sociedade e os aloca em despesas que beneficiam outros além dos que são taxados.

De acordo com Gregory e Borland (1999), existem duas abordagens teóricas principais, as quais se distinguem pelos objetivos dos formuladores de políticas, que buscariam maximizar o bem-estar social ou o interesse próprio.

De acordo com a primeira abordagem, os formuladores de políticas que quei-

\footnotetext{
${ }^{2}$ No caso brasileiro, o Plano Diretor da Reforma do Estado, de 1995, definiu que a política salarial para os servidores públicos federais deveria buscar a redução das disparidades entre as remunerações pagas no setor público e privado para funções semelhantes. A análise contida neste artigo possibilitará, de certa forma, avaliar se os estados também buscaram adotar o mesmo princípio para definir as suas respectivas políticas salariais.
} 
ram maximizar o bem-estar social podem ter objetivos de eficiência ou de equidade nos gastos públicos. O objetivo de eficiência pode implicar na escolha de salários que minimizem os custos de produção, utilizando os valores pagos pelo setor privado como parâmetro, como citado (Fogel e Lewin, 1974; Ehrenberg, 1973; Ashenfelter e Ehrenberg, 1975; Ehrenberg e Goldstein, 1975).

Por outro lado, o objetivo de equidade pode implicar no estabelecimento de um nível de emprego ou de remuneração que vise à correção de imperfeições do mercado de trabalho no qual o governo está inserido, como a discriminação de minorias (Gregory e Borland, 1999). Fogel e Lewin (1974) afirmam que muitos governos definem salários mais altos em relação ao setor privado em razão da existência de um mercado de trabalho privado de monopsônio ou de fatores que impedem a mobilidade, confinando alguns trabalhadores a um mercado "secundário", que corresponderia a uma pequena parte do mercado de trabalho total. Os autores indicam que os defensores desta prática, a qual visa a maior equidade, assumem que os governos provêm os trabalhadores pouco qualificados com melhores compensações do que eles receberiam no setor privado "secundário" na esperança de que o escopo dos esforços do setor público como empregador seja suficiente para aumentar os salários e melhorar as condições nos mercados "secundários".

Já para a segunda abordagem teórica, o objetivo da formulação de políticas não seria a maximização do bem-estar social, mas sim a maximização dos interesses dos próprios formuladores (Gregory e Borland, 1999). De acordo com essa abordagem, políticos estariam preocupados com a maximização de votos, enquanto burocratas teriam o objetivo de maximizar a magnitude do orçamento.

$\mathrm{Na}$ definição dos níveis salariais, mais especificamente, os políticos que buscam a maximização dos votos seriam sensíveis a dois eleitorados: os servidores, afetados diretamente, e o restante do público, afetado indiretamente pelos impostos mas que não tem muita informação sobre esse tipo de política. Políticos seriam mais sensíveis ao primeiro grupo que ao segundo, especialmente pela natureza do processo político envolvido, portanto haveria uma tendência no sentido de os políticos apoiarem as preferências dos servidores (Gregory e Borland, 1999).

Niskanen (1971), por sua vez, foi um dos primeiros a identificar os burocratas como uma categoria dentro do setor público desejando maximizar o tamanho de seu orçamento e capazes de impor suas próprias preferências sobre o partido que governa. Dada a relação assimétrica entre a própria burocracia e os políticos que tomam as decisões (seus financiadores), já que os burocratas possuiriam melhor informação sobre os custos de oferta do produto do que os seus financiadores, os burocratas tentariam maximizar o seu orçamento sujeito à restrição de que o orçamento total tem de ser igual ou maior que os custos de produção. Por outro lado, os políticos tentariam transferir orçamento para os burocratas de forma que eles realizem o produto a um custo mínimo. A solução de equilíbrio resultaria no envio ao Legislativo de um orçamento maior que o custo mínimo necessário, parte dessa diferença poderia ser apropriada na forma de salários - uma das razões pelas quais os burocratas desejariam maximizar o tamanho do orçamento. 
Desta forma, podemos identificar que as políticas salariais adotadas pelo setor público com objetivo de maximização do bem-estar social podem obedecer ao critério de eficiência, ao seguir os níveis remuneratórios praticados pelo setor privado para ocupações semelhantes, ou ao critério de equidade (ou de compensação), buscando compensar remunerações praticadas pelo setor privado e consideradas não adequadas pelos administradores públicos, por meio do estabelecimento de salários mais elevados para os servidores que aqueles pagos pelo setor privado em ocupações semelhantes. Vimos ainda que, além desses critérios, as políticas salariais adotadas podem ter como objetivo a maximização do interesse próprio de políticos e burocratas (apropriação): os níveis salariais seriam estabelecidos acima dos custos de produção, o que equivaleria a dizer que também seriam superiores aos níveis pagos pelo setor privado. No entanto, diferentemente do critério de equidade, este comportamento não dependeria da existência de um mercado de trabalho com imperfeições a serem corrigidas.

\section{Estudos empíricos sobre os diferenciais de salário público-privado}

Smith (1977) realizou um dos primeiros estudos que comparam os salários pagos pelos setores público e privado, através do cálculo do diferencial de salários entre o setor privado e os setores público estadual e federal dos Estados Unidos. Seus principais resultados apontam para um diferencial positivo (prêmio de salário) para o setor público, sendo maior para os servidores federais que para os estaduais. Após esse trabalho, diversos pesquisadores usaram abordagens empíricas alternativas para responder à mesma questão, especialmente para os países desenvolvidos ${ }^{3}$.

Blanchflower (1996) apresenta evidências de prêmios de salário para o setor público de 12 países da OCDE, enquanto Blank (1993) obtém os mesmos resultados para o Reino Unido e para os Estados Unidos. Em ambos os estudos, os resultados para este último país apresentam prêmios no nível federal mas diferenciais negativos (penalidades) para os trabalhadores de governos estaduais e locais, o que também foi apontado por Belman e Heywood (1990) e Moore e Raisian (1991).

Outros trabalhos com a mesma temática demonstram que esses diferenciais não se apresentam de forma geograficamente homogênea. Poterba e Rueben (1998) encontraram grande variância entre os resultados dos diversos estados americanos. Gyourko e Tracy (1991) obtiveram resultados semelhantes ao comparar os salários das áreas metropolitanas americanas.

Nesse sentido, Katz e Krueger (1991) encontram evidências de que os níveis de remuneração de servidores públicos estaduais e locais americanos são consideravelmente sensíveis às condições econômicas regionais - aquelas que afetam o orçamento e a base tributária - enquanto os servidores federais aparentemente não seriam afetados por essas condições, já que seus salários seriam determinados de forma centralizada para todo o país.

\footnotetext{
${ }^{3}$ Sobre os resultados destes estudos, ver Gregory e Borland (1999).
} 
Na mesma linha, Borjas (1986), ao encontrar uma grande variância nos diferenciais entre os salários pagos pelos governos dos 15 maiores estados americanos tomando como base o estado do Texas, rejeita a hipótese de que essa variação nos salários dos servidores públicos refletiria os níveis salariais pagos pelo setor privado nos respectivos estados. Adicionalmente, ele encontra evidências de que mais de $75 \%$ dessa variação poderia ser explicada por variáveis políticas, como a composição do eleitorado e o comportamento do eleitor.

Em se tratando de países em desenvolvimento, existem alguns estudos encontrados com essa temática - nenhum deles usando amostras de governos subnacionais ${ }^{4}$. Um dos estudos mais recentes é o de Panizza e Qiang (2005), que encontrou prêmios de salário para os servidores públicos da maior parte dos 13 países latino-americanos pesquisados, sendo o Brasil um dos que apresentaram os maiores prêmios.

A literatura brasileira a respeito do diferencial de salários entre os setores público e privado também é restrita 5 . Marconi $(2003,2004)$ obtém evidências de que os servidores públicos municipais teriam remunerações semelhantes ou um pouco menores que os trabalhadores do setor privado. Enquanto isso, servidores federais e estaduais receberiam, em média, salários melhores do que se estivessem empregados no setor privado - sendo os diferenciais maiores para os servidores federais. $\mathrm{O}$ autor também calculou o diferencial de salário por nível de governo e escolaridade e demonstrou que o diferencial é positivo para a maioria dos grupos considerados, sendo mais elevado para os servidores com menos anos de estudo, o que vai ao encontro da hipótese de Fogel e Lewin (1974) no sentido de que o governo pode estar preocupado com questões de equidade quando define a política salarial. Barros et al. (2000) comparam os diferenciais entre as regiões metropolitanas, também observando uma significante heterogeneidade de resultados. Os resultados demonstram, dentre outros, que enquanto o setor público do Distrito Federal paga os maiores prêmios de salário, em São Paulo é encontrado um diferencial negativo em relação ao setor privado, dado o desenvolvimento desse setor neste estado.

\section{A MEDIDA DO DIFERENCIAL DE SALÁRIOS PÚBLICO-PRIVADO PARA OS GOVERNOS ESTADUAIS BRASILEIROS}

\section{O cálculo dos diferenciais}

Para verificar a existência de diferenciais de salários entre os trabalhadores dos setores públicos estaduais e dos setores privados nos estados do Brasil, utiliza-

\footnotetext{
${ }^{4}$ Alguns dos estudos encontrados referem-se ao Chile (Corbo e Stelcner, 1983), à Costa Rica (Gindling, 1991) e à Costa do Marfim (Van der Gaag e Vijverberg, 1988).

${ }^{5}$ Macedo (1985), Saldanha et al. (1988), Urani e Ramalho (1995) e Fernandes (2000) são exceções, calculando os diferenciais para diferentes recortes de trabalhadores públicos e privados em diferentes épocas.
} 
remos a técnica desenvolvida por Oaxaca (1973), a qual possibilita decompor tal diferencial de forma a identificar a parcela que é devida às características observáveis dos trabalhadores (como as demográficas, produtivas e institucionais que possam ser associadas a eles) e a relativa às características não observáveis. Como queremos avaliar as práticas salariais nos estados, estaremos interessados em apurar a parcela do diferencial relativa às características não observáveis já tendo controlado as diferenças referentes às características observáveis ${ }^{6}$.

Para tanto, definimos as equações para estimar o salário de trabalhadores do setor público e do setor privado de forma separada, recebendo as notações pub e priv, respectivamente:

$$
\begin{array}{ll}
\operatorname{In}\left(w_{p u b, i}\right)=x_{p u b, i}^{\prime} \beta_{p u b}+\varepsilon_{p u b, i} & i=1, \ldots, n_{p u b} \\
\mathrm{e} & \\
\operatorname{In}\left(w_{p r i v, i}\right)=x_{p \dot{p}, i}^{\prime} \beta_{p r i v}+\varepsilon_{p r i v, i} & i=1, \ldots, n_{p r v}
\end{array}
$$

em que $w_{i}$ é o salário ajustado de acordo com o número de horas trabalhadas para o $i$-ésimo trabalhador;

$x_{i}^{\prime}$ é o vetor com as características observáveis do $i$-ésimo trabalhador;

$\beta$ é o vetor dos coeficientes;

$\varepsilon_{i}$ é o termo aleatório.

Seguindo as mesmas etapas que Greene (2003) ao comparar as duas funções:

$$
\begin{aligned}
& E\left[\ln w_{p u b, i}\right]-E\left[\ln w_{p r i v, i}\right]=x_{p h b i, i}^{\prime} \beta_{p u b}-x_{p h i, i}^{\prime} \beta_{p r i v} \\
& =x_{p w b i}^{\prime} \beta_{p u b}-x_{p w b i, i}^{\prime} \beta_{p r i v}+x_{p w b i, i}^{\prime} \beta_{p r i v}-x_{p r i v i}^{\prime} \beta_{p r i v} \\
& =x_{p u b i, i}^{\prime}\left(\beta_{p u b}-\beta_{p r i v}\right)+\left(x_{p u b, i}-x_{p r i v i, i}\right)^{\prime} \beta_{p r i v}
\end{aligned}
$$

sendo que o primeiro termo dessa decomposição indica o diferencial de salários entre os trabalhadores dos setores público e privado decorrente de características não observáveis - o que buscamos -, e o segundo termo corresponde às diferenças devidas às características observáveis.

O vetor $x_{i}$ de características observáveis incluído nas equações acima é composto das seguintes variáveis: anos de estudo, anos de estudo ao quadrado, idade, idade ao quadrado, experiência no trabalho, experiência no trabalho ao quadrado (todas com o intuito de apurar características produtivas que possam influir no comportamento dos salários); uma dummy para gênero e outra para cor, as quais podem captar a influência de características demográficas que possam ser alvo de discriminação e, desta forma, influir na definição dos níveis salariais; e uma dummy para indicar a filiação do trabalhador a um sindicato, com o intuito de captar a influência de uma variável institucional relevante no processo de formação dos salários (qual seja, o poder de barganha dos trabalhadores). Esta relação

\footnotetext{
${ }^{6}$ Para maiores detalhes sobre a técnica adotada, ver Oaxaca (1973).
} 
de variáveis também é usualmente adotada em outros trabalhos que analisam os diferenciais de salários ${ }^{7}$.

As próximas subseções descreverão os dados utilizados e os testes realizados com o intuito de calcular os diferenciais de salário público-privado segundo a metodologia já descrita.

\section{Os dados utilizados}

Para o cálculo dos diferenciais de salários, a fonte primária de dados foi obtida com a utilização da Pesquisa Nacional por Amostra de Domicílios (PNAD) publicada pelo Instituto Brasileiro de Geografia e Estatística (IBGE). Trabalhamos com os dados dos 26 estados brasileiros e o Distrito Federal, entre os anos de 1995 e $2004^{8}$.

Para o cálculo dos diferenciais de salário incluímos apenas as pessoas empregadas (foram excluídos os trabalhadores por conta própria, os trabalhadores domésticos e os empregadores), com idade entre 18 e 65 anos e em atividades não-agrícolas do país. Esses cortes na amostra geral dos trabalhadores foram necessários para que se pudessem comparar as características do mercado de trabalho público e privado, pois não existem trabalhadores por conta própria ou empregadores no setor público e os concursos e processos de seleção simplificados impõem como requisito de ingresso, em geral, a idade mínima de 18 anos. Adicionalmente, o trabalho em atividades agrícolas possui características específicas que dificultariam sua comparação com os trabalhadores do setor público. Foram também desconsideradas as informações que não explicitavam se o trabalhador atuava no setor público ou privado e incluídas apenas as relativas ao trabalho principal da pessoa, uma vez que a ocupação secundária possui características específicas que poderiam distorcer os resultados.

Todos os valores dos salários individuais foram padronizados em 40 horas de trabalho semanal, estando implícita neste cálculo a suposição de que o valor do salário-hora não se altera de acordo com o número de horas trabalhadas e de que a maioria dos trabalhadores trabalha 40 horas semanais. Foram também desconsideradas as informações relativas aos trabalhadores que tenham informado a prática de uma jornada de trabalho inferior a 20 horas ou superior a 72 horas sema-

\footnotetext{
${ }^{7}$ Exemplos de outros trabalhos que adotam esta formulação para a equação de salários e a relação de variáveis descrita acima no caso brasileiro: Macedo (1985), Barros et al. (2000), Fernandes (2000) e Marconi (2003, 2004).

${ }^{8} \mathrm{O}$ ano 2000 não está contemplado em nossa pesquisa pelo fato de não haver PNAD em anos de realização de Censo. A escolha do período de 1995 a 2004 deu-se pelo objetivo de realizarmos uma análise comparativa num ambiente em que os estados trabalhassem sob uma restrição orçamentária do tipo hard, após o processo de estabilização dos preços e de forte restrição ao financiamento de déficits públicos através do imposto inflacionário. Sobre restrições do tipo soft ou hard, ver Bacha (1994), Stein (1999) e Bevilacqua (2002).
} 
nais, por entendermos que tal situação escapa ao padrão usual e poderia distorcer os nossos resultados.

$\mathrm{Na}$ tentativa de buscarmos maior robustez nos nossos resultados, fizemos testes adicionais considerando amostras distintas de funcionários. Para funcionários públicos trabalhamos com uma amostra considerando todos os funcionários estaduais, incluindo os contratados pelo regime estatutário e pelo regime celetista, por estes dois grupos não apresentarem diferenças significativas nos resultados encontrados de forma separada9 . Para os funcionários privados trabalhamos com duas amostras distintas: uma considerando somente os funcionários do setor privado formal (com registro em carteira) e outra considerando todos os funcionários do setor (mercados formal e informal). O objetivo da inclusão dessas duas amostras é observar se o diferencial permanece mesmo quando consideramos condições de trabalho diversas no setor privado, o que reforçaria nossos resultados. Tal divisão segue a metodologia de análise utilizada em Barros et al. (2000) e Panizza (2001), sendo que o último opta por trabalhar apenas com o setor privado formal. As estatísticas descritivas das variáveis utilizadas em cada uma das amostras encontramse no Apêndice 1 deste trabalho.

Dentre os trabalhadores do setor público, foram excluídos os funcionários das esferas federal e municipal, já que a parcela do setor público considerada nesta pesquisa é composta dos servidores estaduais, englobando aqueles dos Poderes Executivo, Legislativo e Judiciário. Embora acredite-se que a análise das políticas salariais de cada um dos três Poderes seja de extrema relevância, os dados utilizados nesta pesquisa só permitem a segmentação entre eles até o ano de 2001. Assim, a fim de buscar identificar se existe uma diferença significativa entre os níveis remuneratórios praticados nestes Poderes, calculamos os diferenciais de salário para uma amostra contendo apenas os servidores do Poder Executivo dos estados, excluindo aqueles dos Poderes Legislativo e Judiciário para os anos de 1995 a 2001. O objetivo da utilização dessa amostra foi observar se a exclusão desses servidores resultaria em um diferencial menor, demonstrando assim que os diferenciais para os servidores do Legislativo e Judiciário seriam bem maiores que os calculados para os servidores do Poder Executivo, da forma como se imagina, e se tal exclusão poderia alterar a classificação que realizamos. Os diferenciais para os servidores do Executivo dos estados foram, em média, 4 pontos percentuais mais baixos que os dos servidores do setor público geral de cada estado e não alteram a classificação dos padrões de políticas salariais a serem apresentadas ${ }^{10}$.

Os diferenciais de salário público-privado estimados para os diversos estados brasileiros

\footnotetext{
${ }^{9}$ Os resultados que demonstram essa afirmação podem ser solicitados aos autores.

${ }^{10}$ Idem ao anterior. Embora não seja o foco deste trabalho, vale ressaltar que os diferenciais de salário também podem variar de acordo com a carreira dos servidores públicos. Essa abordagem é encontrada no estudo de Moriconi (2007).
} 
Os resultados estimados para o diferencial de salários público-privado nos estados brasileiros estão dispostos na Tabela 1 a seguir:

Tabela 1: Diferencial médio de salários dos funcionários públicos estaduais em relação ao setor privado por região e estado para os anos de 1995 a 2004

\begin{tabular}{|c|c|c|}
\hline $\begin{array}{c}\text { ESTADOS / } \\
\text { REGIÃO }\end{array}$ & Amostra [1] & Amostra [2] \\
\hline AP & $90.1 \%$ & $82.5 \%$ \\
\hline RR & $70.6 \%$ & $53.4 \%$ \\
\hline RO & $35.8 \%$ & $34.6 \%$ \\
\hline AC & $21.0 \%$ & $20.2 \%$ \\
\hline PA & $5.1 \%$ & $14.0 \%$ \\
\hline AM & $1.9 \%$ & $8.2 \%$ \\
\hline NORTE & $37.4 \%$ & $35.5 \%$ \\
\hline MA & $55.1 \%$ & $62.3 \%$ \\
\hline AL & $20.9 \%$ & $25.4 \%$ \\
\hline SE & $18.1 \%$ & $24.0 \%$ \\
\hline BA & $3.3 \%$ & $10.9 \%$ \\
\hline CE & $17.6 \%$ & $28.4 \%$ \\
\hline PI & $12.1 \%$ & $25.2 \%$ \\
\hline PB & $8.4 \%$ & $22.0 \%$ \\
\hline PE & $9.5 \%$ & $16.4 \%$ \\
\hline RN & $0.3 \%$ & $6.3 \%$ \\
\hline NORDESTE & $16.2 \%$ & $24.6 \%$ \\
\hline
\end{tabular}

\begin{tabular}{|c|c|c|}
\hline $\begin{array}{c}\text { ESTADOS / } \\
\text { REGIÃO }\end{array}$ & Amostra [1] & Amostra [2] \\
\hline DF & $38.9 \%$ & $41.1 \%$ \\
\hline TO & $19.7 \%$ & $38.8 \%$ \\
\hline MT & $11.2 \%$ & $17.7 \%$ \\
\hline GO & $5.9 \%$ & $11.1 \%$ \\
\hline MS & $0.5 \%$ & $7.8 \%$ \\
\hline CENTRO-OESTE & $15.3 \%$ & $23.3 \%$ \\
\hline MG & $18.5 \%$ & $22.7 \%$ \\
\hline ES & $15.1 \%$ & $19.9 \%$ \\
\hline RJ & $7.6 \%$ & $9.4 \%$ \\
\hline SP & $-7.2 \%$ & $-5.2 \%$ \\
\hline SUDESTE & $8.5 \%$ & $11.7 \%$ \\
\hline SC & $5.3 \%$ & $6.8 \%$ \\
\hline PR & $2.0 \%$ & $4.9 \%$ \\
\hline RS & $-2.0 \%$ & $2.1 \%$ \\
\hline SUL & $1.8 \%$ & $4.6 \%$ \\
\hline \multicolumn{2}{|c|}{} \\
\hline BRASIL & $18.0 \%$ & $22.6 \%$ \\
\hline
\end{tabular}

Notas: Amostra [1]: Diferencial de salários entre setor público e setor privado formal; Amostra [2]: Diferencial de salários entre setor público e setor privado (formal e informal).

Da mesma forma que a maioria dos governos federais de países analisados em estudos semelhantes apresenta prêmios de salário em relação aos respectivos setores privados, a média geral dos estados brasileiros também aponta para diferenciais de salário público-privado positivos.

Adicionalmente, podemos perceber que os prêmios de salário para o setor público se mantêm em ambas as amostras utilizadas - setor privado formal e setor privado total -, porém são maiores na maioria dos estados quando se considera também o setor informal da economia na amostra. Esta diferença se deve às condições do mercado de trabalho informal, cuja ausência de formalização do contrato de trabalho parece implicar relações de trabalho mais precárias, sujeitas a maior rotatividade e, portanto, também a um menor poder de barganha e possibilidade de organização por parte dos trabalhadores com o intuito de reivindicar melhorias salariais.

Foram encontradas evidências importantes em relação à heterogeneidade de resultados entre os estados brasileiros, sendo que a média dos diferenciais de salário público-privado para o período observado variou de $-7.2 \%$ para São Paulo até +90.1\% para o Amapá, considerando a amostra que exclui o setor privado informal; e de $-5.2 \%$ até $+82.5 \%$, para os mesmos estados, considerando a amostra que possui o conjunto total do setor privado. 
Em relação à disposição regional dos diferenciais público-privado no país, existe uma clara tendência de que o prêmio médio dos salários se concentre nos estados do Norte, Nordeste e Centro-Oeste, enquanto os estados do Sul e Sudeste apresentam prêmios menores ou até mesmo penalidades nos salários recebidos pelos trabalhadores do setor público. Temos, porém, que nos atentar ao comportamento de alguns estados que diferem da média de suas regiões, a exemplo de $\mathrm{Mi}$ nas Gerais e Espírito Santo no Sudeste do país, com consideráveis prêmios de salários dentro de sua região.

Desta forma, podemos afirmar que há fortes evidências de que o setor público brasileiros adota comportamentos diversos nos estados em relação à prática salarial observada nos respectivos setores privados. Para avançar no estudo dos diferenciais e buscar padrões de comportamento dos setores públicos estaduais na definição da remuneração de seus servidores, passaremos à análise dos dados referentes aos diferenciais entre os estados calculados para cada setor.

\section{EXISTEM GRUPOS DE ESTADOS COM O MESMO PADRÃO DE POLÍTICA SALARIAL?}

\section{A metodologia para verificar semelhanças de política salarial}

Com a finalidade de apurarmos se existem grupos de estados com a mesma política salarial, resolvemos comparar todos os salários pagos no setor público e no setor privado com o salário praticado no respectivo setor no estado que apresentou os melhores resultados em termos de eficiência no período analisado. Entendemos que esse procedimento tem dupla vantagem. Primeiro, a partir da referência do estado que buscou uma política de eficiência (o menor diferencial em relação ao setor privado), é possível comparar os demais estados e caracterizar um tipo de política salarial pública. Segundo, o método adotado possibilita avaliar se o diferencial mais favorável ao setor público em um determinado estado se deve ao fato de que o setor privado pratica níveis salariais muito reduzidos.

Nossa escolha foi resultado de duas medidas. Primeiro, do menor diferencial de salários médio encontrado no período analisado, já apresentado na Tabela 2. Segundo, a menor variância ao longo do período analisado, demonstrada no Apêndice 2.

Dada essa definição, escolhemos o estado do Rio Grande do Sul como o estado parâmetro de eficiência. Portanto, utilizando a mesma metodologia apresentada na seção terceira deste trabalho, calcularemos o diferencial entre o setor público estadual do Rio Grande do Sul e cada um dos setores públicos estaduais dos demais estados. No mesmo sentido, estimaremos o diferencial entre o setor privado do Rio Grande do Sul e cada um dos setores privados dos diversos estados. Assim, será possível observar se os diferenciais de salário público-privado encontrados na primeira etapa do trabalho podem ser considerados consequências dos padrões de salários dos respectivos setores privados. 


\section{Classificação da política salarial dos estados}

Tabela 2: Diferencial médio de salários em relação ao estado do Rio Grande do Sul por setor analisado para os anos de 1995 a 2004

\begin{tabular}{|c|c|c|c|}
\hline ESTADOS/REGIÃO & Setor Privado Formal & Setor Privado Total & Setor Público \\
\hline $\mathrm{RR}$ & $8.5 \%$ & $4.9 \%$ & $29.4 \%$ \\
\hline $\mathrm{AP}$ & $0.7 \%$ & $-2.5 \%$ & $42.8 \%$ \\
\hline $\mathrm{RO}$ & $-3.9 \%$ & $-2.0 \%$ & $20.2 \%$ \\
\hline AM & $-3.1 \%$ & $-5.0 \%$ & $1.3 \%$ \\
\hline $\mathrm{AC}$ & $-8.4 \%$ & $-9.8 \%$ & $15.7 \%$ \\
\hline PA & $-17.5 \%$ & $-20.6 \%$ & $-15.0 \%$ \\
\hline NORTE & $-4.0 \%$ & $-5.8 \%$ & $15.7 \%$ \\
\hline BA & $-19.0 \%$ & $-23.0 \%$ & $-16.1 \%$ \\
\hline $\mathrm{AL}$ & $-24.5 \%$ & $-26.5 \%$ & $-12.7 \%$ \\
\hline SE & $-24.7 \%$ & $-25.3 \%$ & $-12.9 \%$ \\
\hline PE & $-25.4 \%$ & $-27.7 \%$ & $-18.9 \%$ \\
\hline MA & $-26.7 \%$ & $-37.4 \%$ & $-13.6 \%$ \\
\hline $\mathrm{CE}$ & $-26.8 \%$ & $-28.9 \%$ & $-13.4 \%$ \\
\hline PB & $-28.2 \%$ & $-30.9 \%$ & $-26.8 \%$ \\
\hline $\mathrm{RN}$ & $-28.8 \%$ & $-31.4 \%$ & $-36.9 \%$ \\
\hline $\mathrm{PI}$ & $-31.2 \%$ & $-37.3 \%$ & $-30.3 \%$ \\
\hline NORDESTE & $-26.1 \%$ & $-29.8 \%$ & $-20.2 \%$ \\
\hline DF & $19.5 \%$ & $21.6 \%$ & $77.8 \%$ \\
\hline MT & $1.7 \%$ & $1.5 \%$ & $8.7 \%$ \\
\hline $\mathrm{GO}$ & $-9.3 \%$ & $-10.0 \%$ & $-11.4 \%$ \\
\hline MS & $-9.8 \%$ & $-10.6 \%$ & $-9.6 \%$ \\
\hline TO & $-12.2 \%$ & $-20.7 \%$ & $-8.7 \%$ \\
\hline CENTRO-OESTE & $-2.0 \%$ & $-3.7 \%$ & $11.4 \%$ \\
\hline $\mathrm{SP}$ & $27.3 \%$ & $27.8 \%$ & $24.0 \%$ \\
\hline RJ & $-1.5 \%$ & $0.4 \%$ & $5.0 \%$ \\
\hline ES & $-7.5 \%$ & $-8.3 \%$ & $12.3 \%$ \\
\hline MG & $-9.6 \%$ & $-10.2 \%$ & $5.5 \%$ \\
\hline SUDESTE & $2.2 \%$ & $2.4 \%$ & $11.7 \%$ \\
\hline $\mathrm{SC}$ & $6.8 \%$ & $9.6 \%$ & $8.3 \%$ \\
\hline PR & $1.6 \%$ & $1.9 \%$ & $5.8 \%$ \\
\hline $\mathrm{RS}$ & $0.0 \%$ & $0.0 \%$ & $0.0 \%$ \\
\hline SUL & $2.8 \%$ & $3.8 \%$ & $4.7 \%$ \\
\hline BRASIL & $-9.3 \%$ & $-11.1 \%$ & $1.1 \%$ \\
\hline
\end{tabular}

Notas: Setor Privado Formal: Diferencial de salários entre os setores privados formais do estado indicado e do Rio Grande do Sul. Setor Privado Total: Diferencial de salários entre os setores privados totais (formais e informais) do estado indicado e do Rio Grande do Sul. Setor Público: Diferencial de salários entre os setores públicos do estado indicado e o setor público do Rio Grande do Sul.

$\mathrm{Na}$ tabela apresentada, podemos visualizar que a remuneração privada no mercado de trabalho do Rio Grande do Sul está entre as maiores do país, dado 
que apenas sete estados apresentaram setores privados com salários médios superiores no período analisado, quando analisados os trabalhadores com registro em carteira ou aqueles do setor privado total.

Analisando os resultados relativos ao setor público, é possível verificar que os setores públicos estaduais de 13 estados brasileiros praticam remunerações médias superiores às observadas no setor público estadual do Rio Grande do Sul, os quais se concentram nas regiões Sul, Sudeste e Norte (com exceção do Pará), estando o prêmio presente também nos salários no Distrito Federal e em Mato Grosso (região Centro-Oeste). Nenhum estado da região Nordeste apresentou diferencial de remuneração do setor público positivo em relação aos valores praticados no Rio Grande do Sul.

Assim como a heterogeneidade encontrada nos resultados do cálculo dos diferenciais público-privado, os resultados desta segunda etapa do trabalho também apontam para a diversidade de políticas salariais adotadas pelos setores públicos estaduais ${ }^{11}$. A partir dos dados demonstrados na Tabela 2, optamos por criar três categorias de classificação ${ }^{12}$ para o comportamento dos estados em relação à conduta de suas políticas salariais, tomando como parâmetro o respectivo setor privado:

Categoria (I) Eficiência: composta por estados cujos setores públicos apresentam diferenciais de salário em relação ao Rio Grande do Sul iguais ou próximos àqueles apresentados pelos respectivos setores privados na mesma base de comparação. Este resultado indicaria que o setor público estadual acompanharia os níveis salariais praticados pelo respectivo setor privado - uma política salarial que pode ser considerada eficiente, pois minimizaria os custos de produção ao governo estadual.

Categoria (II) Compensação: composta por estados cujo setor público apresenta prêmio de salário em relação aos valores observados no setor público no Rio Grande do Sul enquanto o respectivo setor privado apresenta penalidades salariais em relação ao setor privado do Rio Grande do Sul. Este resultado demonstraria que o setor público estadual buscaria pagar salários mais elevados que o respectivo setor privado para compensar baixos níveis de remuneração praticados neste último. Neste caso, há evidências de que o diferencial pode ser uma consequência do comportamento do setor privado estadual.

\footnotetext{
${ }^{11}$ Embora tenhamos obtido os diferenciais referentes a cada ano da amostra, optamos pela utilização das médias dos resultados no período para possibilitar analisar se houve um padrão de políticas salariais praticadas pelos estados no intervalo considerado. Apesar da significativa variação desses resultados ao longo do período, as evidências apontam para melhorias na remuneração dos servidores estaduais em frente à dos empregados do setor privado em todos os estados nos dois últimos anos da amostra. Assim sendo, em havendo dados disponíveis, será de extrema relevância a análise de períodos posteriores.

${ }^{12}$ Classificação baseada nos argumentos trabalhados em Niskanen (1971), Ehrenberg (1973), Fogel e Lewin (1974), Ashenfelter e Ehrenberg (1975), Ehrenberg e Goldstein (1975) e Gregory e Borland (1999).
} 
Categoria (III) Apropriação: composta por estados cujo setor público apresenta prêmios de salário em relação ao setor público no Rio Grande do Sul maiores que aqueles que o respectivo setor privado apresenta em relação ao setor privado no estado de controle (RS). Neste caso, os níveis de salário no setor público seriam mantidos acima dos níveis de eficiência sem justificativas de busca de compensação em relação ao setor privado e tenderíamos a apontar a presença de um comportamento de apropriação de recursos nas decisões relativas à política salarial.

Uma vez que estamos trabalhando com a média dos diferenciais de salários estimados nos nove anos de análise, uma classificação mais precisa dos estados nas categorias estipuladas deve levar em consideração uma eventual margem de erro das estimações. Para identificarmos se os diferenciais de salário para o setor público de cada estado em relação ao Rio Grande do Sul podem ser realmente considerados diferentes daqueles apresentados pelos respectivos setores privados, foram feitos testes de diferença de médias, considerando um nível de $10 \%$ de significância - cujos resultados encontram-se no Apêndice $3 .{ }^{13}{ }^{14}$ A partir destes testes, pudemos realizar a seguinte classificação dos estados nas três categorias:

Tabela 3: Classificação dos estados brasileiros seguindo dinâmica salarial do setor público (considerando teste de médias com significância de 10\%)

\begin{tabular}{|l|l|}
\hline Categoria & Estados \\
\hline \multirow{3}{*}{ (I) Eficiência } & $\begin{array}{l}\text { Goiás, Mato Grosso do Sul, Mato Grosso, Amazonas, } \\
\text { Pará, Paraíba, Paraná, Santa Catarina, Rio de Janeiro e } \\
\text { São Paulo. }\end{array}$ \\
\hline (II) Compensação & $\begin{array}{l}\text { Tocantins, Acre, Amapá, Rondônia, Alagoas, Bahia, Ceará, } \\
\text { Maranhão, Pernambuco, Piauí, Rio Grande do Norte, } \\
\text { Sergipe, Espírito Santo e Minas Gerais. }\end{array}$ \\
\hline (III) Apropriação & Distrito Federal e Roraima. \\
\hline
\end{tabular}

O Gráfico 1 na sequência permite visualizar de forma mais clara a classificação dos estados brasileiros nas categorias acima propostas. Para identificá-los, traçamos uma reta perpendicular aos eixos "Remuneração Setor Privado" e "Remuneração Setor Público Estadual”. Os estados junto à reta correspondem àqueles em que o diferencial de salários praticado no setor público é próximo ao pratica-

\footnotetext{
${ }^{13}$ Os testes estimaram intervalos de confiança a partir dos desvios-padrão calculados com o nível de significância de $10 \%$ e $5 \%$. Assim, os resultados considerados estatisticamente diferentes, segundo este critério e os testes $t$ decorrentes, não foram considerados eficientes.

${ }^{14}$ As únicas alterações em relação à tabela apresentada e os resultados ao nível de $5 \%$ são os estados de Alagoas, Rio Grande do Norte, Sergipe, Pará e Minas Gerais, os quais passaram da Categoria II Compensação para a Categoria I - Eficiência. Tal mudança acontece por não podermos apontar diferenças entre as médias de remuneração dos setores privado e público.
} 
do no setor privado (sempre em comparação ao estado de controle, o Rio Grande do Sul), o que significa que os setores públicos estaduais tendem a oferecer remunerações próximas ao nível definido como eficiente, sendo sua política salarial classificada na Categoria I - Eficiência.

Estados situados acima da reta apresentam diferenciais de salários no setor público maiores que os apresentados no setor privado e podem estar classificados tanto na Categoria II - Compensação como na Categoria III - Apropriação, dependendo do quadrante em que estejam situados.

Se o estado se encontrar no quadrante II, o diferencial de salários de seu respectivo setor privado em relação ao observado no Rio Grande do Sul é negativo e, como o diferencial de salários de seu respectivo setor público em relação ao observado no Rio Grande do Sul é positivo, podemos classificar a política salarial praticada pelo setor público em tal estado como compensatória, pertencendo à Categoria II - Compensação.

Por sua vez, se o estado se encontrar no quadrante III, o diferencial de salários de seu respectivo setor privado em relação ao observado no Rio Grande do Sul é positivo e o diferencial de salários de seu respectivo setor público em relação ao observado no Rio Grande do Sul também é positivo e, adicionalmente, maior que o observado no setor privado. Conforme explicado acima, os estados que apresentam este comportamento em relação às suas práticas salariais estão classificados na Categoria III - Apropriação. Esta classificação é reforçada pelos testes de diferenças de médias realizados.

Destaca-se o fato de que não existe nenhum estado situado abaixo da reta perpendicular aos eixos cujos diferenciais possam ser classificados como estatisticamente distintos ${ }^{15}$. Isso significa que não há estado que apresente um diferencial de salários no setor público (comparado com o setor público do Rio Grande do Sul) inferior ao diferencial de salários encontrado para o setor privado (em relação ao setor privado no Rio Grande do Sul). Não há, portanto, setor público estadual que pratique política salarial que resulte em remunerações inferiores aos patamares definidos como eficientes.

\footnotetext{
${ }^{15}$ Apesar do Rio Grande do Norte estar situado abaixo da curva, os testes de diferenças de médias apontaram que os diferenciais encontrados para este estado não são estatisticamente distintos dado o nível de significância considerado.
} 
Gráfico 1: Relação de salários médios pagos pelo setor público estadual e pelo setor privado entre estados. Diferenciais em referência ao estado do Rio Grande do Sul para os anos de 1995 a 2004

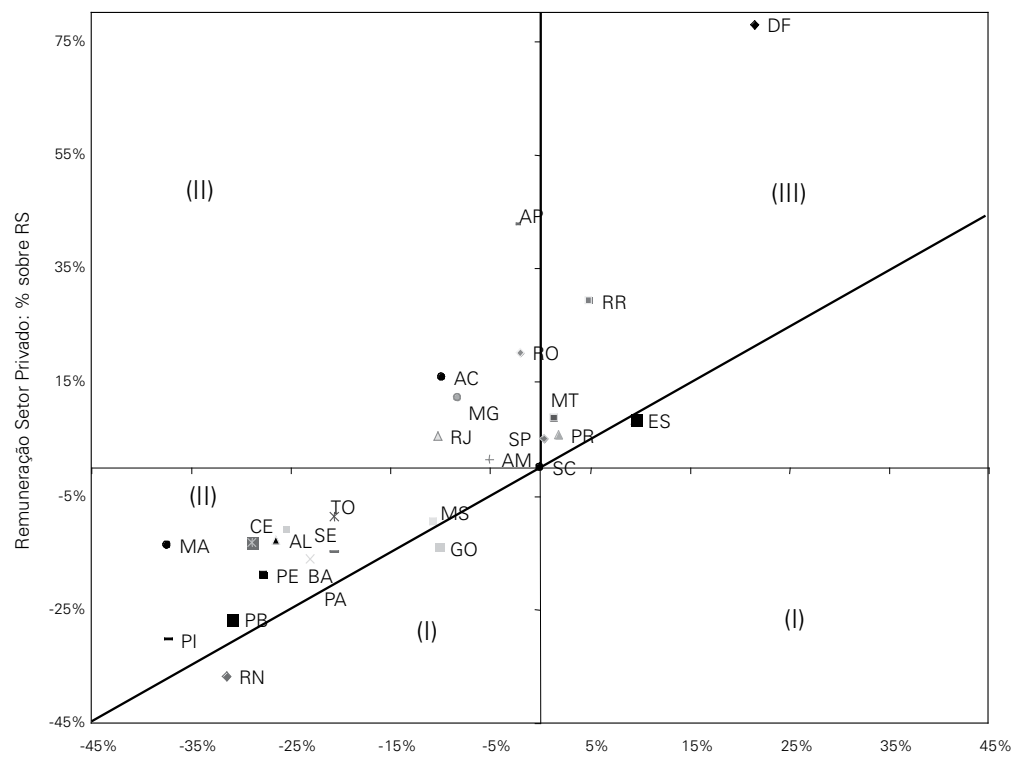

Os estados classificados na categoria Eficiência foram aqueles em que não pudemos apontar diferenças estatisticamente significantes entre as médias dos diferenciais do setor privado e setor público comparativamente à política salarial do Rio Grande do Sul. Dos 13 estados ali classificados, destaca-se a totalidade da região Sul do país, acompanhada pelos estados de São Paulo e Rio de Janeiro na região Sudeste; Goiás, Mato Grosso e Mato Grosso do Sul da região Centro-Oeste; Amazonas e Pará na região Norte e a Paraíba, único representante da região Nordeste do Brasil.

A categoria Compensação é a mais heterogênea, agregando estados cujos setores privados pagam remunerações significantemente baixas (em relação ao Rio Grande do Sul), principalmente na região Nordeste, e outros estados, principalmente da região Norte, nos quais essas remunerações, apesar de comparativamente negativas, não são tão inferiores às pagas no estado de controle. Em ambos os casos, os respectivos setores públicos apresentam prêmios de salários em relação aos respectivos setores privados. Em razão desta grande variabilidade, considera-se a possibilidade de também estar ocorrendo, em certa medida, comportamentos de apropriação de recursos na forma de salários nestes últimos estados.

$\mathrm{Na}$ categoria Apropriação, os estados do Distrito Federal e Roraima apresentam simultaneamente diferenciais positivos em relação ao Rio de Grande do Sul no setor privado $(+21.5 \%$ e $+4.9 \%$, respectivamente $)$ e maior ainda no setor público $(+77.7 \%$ e $+29.4 \%)$.

Assim, os dados e os critérios estatísticos adotados permitem afirmar que existe um número praticamente igual de estados que praticam políticas eficientes e compensatórias e apenas dois estados sobre os quais podemos afirmar, com maior grau 
de segurança, que praticam políticas classificadas como de apropriação de recursos públicos. De toda forma, os resultados encontrados para os estados brasileiros são bastante heterogêneos, indicando comportamento semelhante ao observado em estudos realizados sobre os salários em governos subnacionais em outros países.

\section{CONCLUSÕES}

O estudo aqui apresentado demonstrou que existem diferenciais de salário entre o setor público e privado em vários estados brasileiros e, mais que isso, os diferenciais observados não são uniformes, a exemplo dos resultados encontrados para governos subnacionais de outros países. Dada esta heterogeneidade, podemos afirmar que os setores públicos dos estados possuem práticas salariais distintas.

Demonstramos que, em parte dos estados nos quais foram observados diferenciais positivos, o setor público parece praticar políticas compensatórias, visando à melhoria das remunerações pagas no setor público em relação àquelas pagas no setor privado naquele estado, pois o patamar médio destas últimas seria reduzido em comparação ao nível praticado no estado de referência. Em outros estados, ainda que em número menor, ficou caracterizada uma prática de apropriação de recursos públicos, já que o setor privado remunera seus trabalhadores com valores superiores aos observados no mercado privado em outros estados e, mesmo assim, o setor público oferece remunerações superiores às observadas no setor privado. Por seu turno, alguns estados parecem estar perseguindo os níveis remuneratórios praticados no setor privado daquela localidade, configurando um indicador de uma prática salarial eficiente.

É interessante ressaltar que o grupo caracterizado como eficiente, segundo a definição e os critérios estatísticos adotados nos cálculos, é maior do que o senso comum poderia supor, porém menor que o desejado pela sociedade. O grupo caracterizado como compensatório se concentra nos estados do Nordeste e do Norte, enquanto são poucos os estados que claramente praticam uma política de apropriação de recursos. Há, entretanto, alguns outros estados classificados no grupo que visa à compensação cuja política também pode apresentar traços de um comportamento de apropriação de recursos: o diferencial de salários entre o setor privado nesses estados e no Rio Grande do Sul não é muito distante de zero e o diferencial entre o setor público nesses estados e no Rio Grande do Sul é positivo; esses estados estão localizados principalmente na região Norte.

Destaca-se também o fato de que não existe nenhum estado em que, considerando a significância estatística adotada, tenha sido observado um diferencial de salários entre o setor público no estado e no Rio Grande do Sul inferior ao diferencial de salários entre o setor privado no estado e no Rio Grande do Sul. Assim, apesar de praticarem políticas heterogêneas, há um traço comum entre as estratégias dos estados, qual seja, a adoção de salários que, no mínimo, acompanhem os níveis de remuneração observados nos mercados de trabalho privados locais.

Por fim, entendemos que a heterogeneidade entre os resultados observados 
também pode estar condicionada a outros fatores políticos ou econômicos. Além disso, estas políticas não produzem efeitos apenas dentro do próprio setor público e podem gerar impactos sobre a economia local. Estes constituem uma agenda importante para futuros estudos sobre o tema.

\section{REFERÊNCIAS BIBLIOGRÁFICAS}

ASHENFELTER, O.C.; Ehrenberg, R.G. (1975) “The demand for labor in the public sector”. In: D. Hamermesh, ed., Labor in the Public and Nonprofit Sectors, pp. 55-84. Princeton University Press.

BACHA, E. (1994) “O fisco e a inflação: uma interpretação do caso brasileiro". Revista de Economia Política, 14 (1): 5-17.

BARROS, R.P.; FOGUEL, M.N.; GILL, I.; MENDONÇA, R. (2000) “The public-private wage gap in Brazil”. Revista Brasileira de Economia, 54 (4): 433-472.

Belman, D.; Heywood, J.S. (1990) “The effect of stablishment and firm size on public wage differentials", Public Finance Quarterly, 18:221-235.

BEVILACQUA, A.S. (2002) "State Government Bailouts in Brazil". Working paper of Inter-American Development Bank.

BLANCHFLOWER, D.G. (1996) “The role and influence of unions in the OECD”. Darmouth College, mimeo.

BLANK, R. (1993) "Public sector growth and labor market flexibility: The United States vs. The United Kingdom”. National Bureau of Economic Research. Working paper 4339.

BORJAS, G.J. (1986) “The Earnings of State Government Employees in the United States”, Journal of Urban Economics, 19: 156-173.

CORBO, V; STELCNER, M. (1983) “Earning Determination and Labor Markets: Gran Santiago.” Journal of Development Economics, 12: 251-266.

EHRENBERG, R.G. (1973) “The demand for state and local government employees”, American Economic Review, 63, pp. 366-379.

EHRENBERG R.G.; GOLDSTEIN, G.S. (1975) “A model of public sector wage determination”, Journal of Urban Economics, 2: 223-245.

FERNANDES, R. (2000) “Desigualdade Salarial: Aspectos Teóricos”. São Paulo: Universidade de São Paulo, mimeo.

FOGEL, W.; LEWIN, D. (1974) “Wage Determination in the Public Sector", Industrial and Labor Relations Review, 27 (3): 410-431.

GINDLING, T. (1991) "Labor Market Segmentation and the Determination of Wages in the Public, Private-Formal, and Informal Sectors in San Jose, Costa Rica." Economic Development and Cultural Change, 13: 585-605.

GREGORY, R.G.; BORLAND, J. (1999) “Recent Developments in Public Sector Labor Markets”, in Ashenfelter, O. e D. Card, eds., Handbook of Labor Economics, Vol. 3, cap. 53: 3573-3630. Elsevier/North-Holland.

GREENE, W.H. (2003) Econometric Analysis. $5^{\text {th }}$ Edition, Prentice Hall.

GYOURKO, J.; Tracy, J. (1991) "Public sector bargaining and the local budgetary process”. In: R. Ehrenberg, ed., Research in Labor Economics, 12: 117-136.

KATZ, L.F. e KRUEGER, A.B. (1991) "Changes in the structure of wages in the public and private sectors”. In: R. Ehrenberg, ed., Research in Labor Economics, 12: 131-172.

MACEDO, R. (1985) "Diferenciais de salários entre empresas privadas e estatais no Brasil". Revista Brasileira de Economia, 39 (4): 437-448.

MARCONI, N. (2003) "A evolução do perfil da força de trabalho e das remunerações nos setores público e privado ao longo da década de 1990". Revista do Serviço Público, ano 54, n. 1: 6-42.

MARCONI, N. (2004) "Gap between public and private wages and wages determination in the public sector”. Revista de Economia Política, 24 (2): 257-279.

MOORE, W. J. e RAISIAN, J. (1991) “Government wage differentials revisited". Journal of Labor Research, 12: 13-33. 
MORICONI, G. M. (2007) “Todos os servidores públicos são bem remunerados? Uma comparação entre as carreiras dos governos estaduais brasileiros". Anais do XXXV Encontro Nacional de Economia - ANPEC, Recife-PE.

NISKANEN, W.A. (1971) Bureaucracy and representative government. Chicago: Aldine-Atherton.

OAXACA, R. (1973) "Male-female wage differentials in urban labor markets". International Economic Review, 140: 693-709.

PANIZZA, U.; QIANG, C.Z. (2005) "Public-private wage differential and gender gap in Latin America: Spoiled bureaucrats and exploited women?” The Journal of Socio-Economics, 34: 810-833.

POTERBA, J.; RUEBEN, K. (1998) "Fiscal institutions and public sector labor market. National Bureau of Economic Research". Working Paper 6659.

SALDANHA, R.; MAIA, R.; CAMARGO, J.M. (1988) “Emprego e salário no setor público federal”. Texto para Discussão, 5. Brasília, Ministério do Trabalho.

SMITH, S. (1977) “Government Wage Differentials”. Journal of Urban Economics, 4: 248-271.

STEIN, E. (1999) "Fiscal decentralization and Government size in Latin America". Journal of Applied Economics. Vol II, n. 2: 357-391.

URANI, A.; RAMALHO, M. (1995) “A Administração Pública como Empregadora: Uma Avaliação da Década de 80”. Série Seminários, 5/95. Rio de Janeiro, Ipea.

Van der Gaag, J.; Vijverberg, W. (1988) "A Switching Regression Model for Wage Determinants in the Public and Private Sectors for a Developing Country." Review of Economics and Statistics, 70, pp. 244-252.

\section{Apêndice 1}

Tabela A.1.1: Estatística descritiva dos dados referentes ao setor privado

\begin{tabular}{|c|c|c|c|c|c|c|c|c|}
\hline & \multicolumn{4}{|c|}{$\begin{array}{c}\text { Trabalhadores do Setor Privado } \\
\text { Formal }\end{array}$} & \multicolumn{4}{|c|}{$\begin{array}{l}\text { Trabalhadores do Setor Privado Total } \\
\text { (Formal e Informal) }\end{array}$} \\
\hline Variáveis & Média & $\begin{array}{l}\text { Desvio- } \\
\text {-padrão }\end{array}$ & Mínimo & Máximo & Média & $\begin{array}{l}\text { Desvio- } \\
\text { padrão }\end{array}$ & Mínimo & Máximo \\
\hline \multicolumn{9}{|c|}{ Variável Dependente } \\
\hline $\begin{array}{l}\text { Ln do salário } \\
\text { por hora }\end{array}$ & 2.08 & 0.82 & -1.74 & 6.72 & 2.01 & 0.84 & -1.89 & 6.72 \\
\hline \multicolumn{9}{|c|}{ Variáveis Independentes } \\
\hline Idade & 33.36 & 10.77 & 18 & 65 & 33.03 & 11.00 & 18 & 65 \\
\hline Idade ao quadrado & 1229.66 & 819.82 & 324 & 4225 & 1212.26 & 839.07 & 324 & 4225 \\
\hline Anos de estudo & 8.76 & 4.05 & 1 & 16 & 8.56 & 4.09 & 1 & 16 \\
\hline $\begin{array}{l}\text { Anos de estudo ao } \\
\text { quadrado }\end{array}$ & 93.20 & 72.29 & 1 & 256 & 90.04 & 71.93 & 1 & 256 \\
\hline Anos de Experiência & 4.89 & 6.13 & 0 & 65 & 4.58 & 6.01 & 0 & 65 \\
\hline $\begin{array}{l}\text { Anos de Experiência } \\
\text { ao quadrado }\end{array}$ & 61.55 & 147.01 & 0 & 4225 & 57.18 & 144.67 & 0 & 4225 \\
\hline $\begin{array}{l}\text { Dummy de Gênero } \\
\text { (masculino }=1)\end{array}$ & 0.65 & 0.47 & 0 & 1 & 0.65 & 0.47 & 0 & 1 \\
\hline $\begin{array}{l}\text { Dummy de Cor } \\
(\text { branco }=1)\end{array}$ & 0.57 & 0.49 & 0 & 1 & 0.56 & 0.49 & 0 & 1 \\
\hline $\begin{array}{c}\text { Dummy de } \\
\text { Sindicalização (sin- } \\
\text { dicalizado }=1 \text { ) }\end{array}$ & 0.27 & 0.44 & 0 & 1 & 0.23 & 0.42 & 0 & 1 \\
\hline
\end{tabular}


Tabela A.1.2 Estatística descritiva dos dados referentes ao setor público estadual

\begin{tabular}{|l|c|c|c|c|}
\hline \multicolumn{1}{|c|}{ Variáveis } & Média & $\begin{array}{c}\text { Desvio- } \\
- \text { padrão }\end{array}$ & Mínimo & Máximo \\
\hline Variável Dependente & & & & \\
\hline Ln do salário por hora & 2.64 & 0.82 & -1.56 & 6.90 \\
\hline Variáveis Independentes & & & & \\
\hline Idade & 38.94 & 9.78 & 18 & 65 \\
\hline Idade ao quadrado & 1612.75 & 807.43 & 324 & 4225 \\
\hline Anos de estudo & 12.10 & 3.70 & 1 & 16 \\
\hline Anos de estudo ao quadrado & 160.29 & 78.50837 & 1 & 256 \\
\hline Anos de Experiência & 11.65 & 7.75 & 0 & 49 \\
\hline Anos de Experiência ao quadrado & 195.84 & 226.79 & 0 & 2401 \\
\hline Dummy de Gênero (masculino =1) & 0.41 & 0.49 & 0 & 1 \\
\hline Dummy de Cor (branco = 1) & 0.57 & 0.49 & 0 & 1 \\
\hline $\begin{array}{l}\text { Dummy de Sindicalização } \quad \text { (sin- } \\
\text { dicalizado = 1) }\end{array}$ & 0.38 & 0.48 & 0 & 1 \\
\hline
\end{tabular}

\section{Apêndice 2}

Tabela A.2: Variância dos diferenciais estimados ao longo do período analisado (1995 a 2004)

\begin{tabular}{|c|c|c|}
\hline Estados & Amostra [1] & Amostra [2] \\
\hline AC & $2.64 \%$ & $3.99 \%$ \\
\hline AL & $3.82 \%$ & $3.95 \%$ \\
\hline AM & $1.20 \%$ & $1.02 \%$ \\
\hline AP & $4.73 \%$ & $9.58 \%$ \\
\hline BA & $0.61 \%$ & $0.30 \%$ \\
\hline CE & $2.45 \%$ & $1.30 \%$ \\
\hline DF & $1.19 \%$ & $1.13 \%$ \\
\hline ES & $1.70 \%$ & $1.57 \%$ \\
\hline GO & $1.15 \%$ & $1.08 \%$ \\
\hline MA & $5.73 \%$ & $7.87 \%$ \\
\hline MG & $1.49 \%$ & $1.44 \%$ \\
\hline MS & $1.91 \%$ & $1.91 \%$ \\
\hline MT & $1.53 \%$ & $1.39 \%$ \\
\hline PA & $0.74 \%$ & $1.00 \%$ \\
\hline
\end{tabular}

\begin{tabular}{|c|c|c|}
\hline Estados & Amostra [1] & Amostra [2] \\
\hline $\mathrm{PB}$ & $3.29 \%$ & $1.08 \%$ \\
\hline $\mathrm{PE}$ & $0.24 \%$ & $0.27 \%$ \\
\hline $\mathrm{PI}$ & $2.26 \%$ & $1.55 \%$ \\
\hline $\mathrm{PR}$ & $0.50 \%$ & $0.45 \%$ \\
\hline $\mathrm{RJ}$ & $1.41 \%$ & $1.37 \%$ \\
\hline $\mathrm{RN}$ & $1.09 \%$ & $1.10 \%$ \\
\hline $\mathrm{RO}$ & $2.95 \%$ & $12.28 \%$ \\
\hline $\mathrm{RR}$ & $3.85 \%$ & $23.96 \%$ \\
\hline $\mathrm{RS}$ & $0.48 \%$ & $0.37 \%$ \\
\hline $\mathrm{SC}$ & $0.58 \%$ & $0.59 \%$ \\
\hline $\mathrm{SE}$ & $2.30 \%$ & $1.72 \%$ \\
\hline $\mathrm{SP}$ & $0.59 \%$ & $0.51 \%$ \\
\hline TO & $2.04 \%$ & $1.69 \%$ \\
\hline
\end{tabular}




\section{Apêndice 3}

Tabela A. 3: Intervalo de confiança dos diferenciais estimados

\begin{tabular}{|c|c|c|c|c|c|c|c|c|c|}
\hline \multirow{3}{*}{ Região } & \multirow{3}{*}{ Estados } & \multicolumn{4}{|c|}{ Com $10 \%$ de significância } & \multicolumn{4}{|c|}{ Com $5 \%$ de significância } \\
\hline & & \multicolumn{2}{|c|}{ PRIVADO } & \multicolumn{2}{|c|}{ PÚBLICO } & \multicolumn{2}{|c|}{ PRIVADO } & \multicolumn{2}{|c|}{ PÚBLICO } \\
\hline & & $\begin{array}{l}\text { Média } \\
\text { - I.C. }\end{array}$ & $\begin{array}{l}\text { Média } \\
\text { + I.C. }\end{array}$ & $\begin{array}{l}\text { Média } \\
\text { - I.C. }\end{array}$ & $\begin{array}{l}\text { Média } \\
+ \text { I.C. }\end{array}$ & $\begin{array}{l}\text { Média } \\
\text { - I.C. }\end{array}$ & $\begin{array}{l}\text { Média } \\
+ \text { I.C. }\end{array}$ & $\begin{array}{l}\text { Média } \\
\text { - I.C. }\end{array}$ & $\begin{array}{l}\text { Média } \\
+ \text { I.C. }\end{array}$ \\
\hline \multirow{5}{*}{ 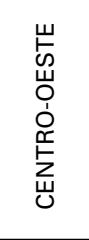 } & DF & $16.2 \%$ & $40.0 \%$ & $14.0 \%$ & $42.3 \%$ & $62.1 \%$ & $87.7 \%$ & $64.2 \%$ & $85.6 \%$ \\
\hline & $\mathrm{GO}$ & $-14.8 \%$ & $-8.1 \%$ & $-15.4 \%$ & $-7.4 \%$ & $-21.5 \%$ & $-3.5 \%$ & $-20.1 \%$ & $-4.9 \%$ \\
\hline & MS & $-15.1 \%$ & $-11.1 \%$ & $-15.4 \%$ & $-10.8 \%$ & $-21.1 \%$ & $-9.3 \%$ & $-20.2 \%$ & $-10.2 \%$ \\
\hline & MT & $-4.8 \%$ & $1.3 \%$ & $-5.4 \%$ & $1.9 \%$ & $-1.8 \%$ & $11.0 \%$ & $-0.8 \%$ & $9.9 \%$ \\
\hline & TO & $-21.6 \%$ & $-12.5 \%$ & $-22.5 \%$ & $-11.6 \%$ & $-18.7 \%$ & $-8.1 \%$ & $-17.8 \%$ & $-9.0 \%$ \\
\hline \multirow{9}{*}{ 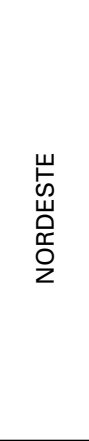 } & $A L$ & $-32.1 \%$ & $-27.9 \%$ & $-32.5 \%$ & $-27.5 \%$ & $-25.6 \%$ & $-14.8 \%$ & $-24.8 \%$ & $-15.7 \%$ \\
\hline & BA & $-25.7 \%$ & $-7.8 \%$ & $-27.4 \%$ & $-6.1 \%$ & $-23.3 \%$ & $-7.1 \%$ & $-22.0 \%$ & $-8.4 \%$ \\
\hline & CE & $-31.4 \%$ & $-28.6 \%$ & $-31.6 \%$ & $-28.3 \%$ & $-22.5 \%$ & $-16.4 \%$ & $-22.0 \%$ & $-16.9 \%$ \\
\hline & MA & $-38.5 \%$ & $-31.8 \%$ & $-39.2 \%$ & $-31.1 \%$ & $-25.3 \%$ & $-13.1 \%$ & $-24.3 \%$ & $-14.0 \%$ \\
\hline & PB & $-37.1 \%$ & $-33.0 \%$ & $-37.5 \%$ & $-32.6 \%$ & $-40.3 \%$ & $-24.1 \%$ & $-39.0 \%$ & $-25.4 \%$ \\
\hline & PE & $-32.1 \%$ & $-3.5 \%$ & $-34.8 \%$ & $-0.7 \%$ & $-24.9 \%$ & $-5.3 \%$ & $-23.3 \%$ & $-6.9 \%$ \\
\hline & PI & $-40.9 \%$ & $-37.3 \%$ & $-41.3 \%$ & $-36.9 \%$ & $-41.8 \%$ & $-29.4 \%$ & $-40.8 \%$ & $-30.4 \%$ \\
\hline & RN & $-36.4 \%$ & $-32.3 \%$ & $-36.8 \%$ & $-31.9 \%$ & $-51.3 \%$ & $-36.7 \%$ & $-50.1 \%$ & $-37.9 \%$ \\
\hline & SE & $-30.8 \%$ & $-21.4 \%$ & $-31.7 \%$ & $-20.4 \%$ & $-22.1 \%$ & $-11.2 \%$ & $-21.2 \%$ & $-12.1 \%$ \\
\hline \multirow{6}{*}{$\begin{array}{l}\frac{w}{\sigma} \\
\text { or } \\
z\end{array}$} & $A C$ & $-14.0 \%$ & $-1.3 \%$ & $-15.2 \%$ & $0.0 \%$ & $0.3 \%$ & $16.1 \%$ & $1.6 \%$ & $14.9 \%$ \\
\hline & AM & $-10.8 \%$ & $-2.4 \%$ & $-11.6 \%$ & $-1.6 \%$ & $-9.6 \%$ & $1.2 \%$ & $-8.7 \%$ & $0.4 \%$ \\
\hline & AP & $-8.9 \%$ & $2.2 \%$ & $-9.9 \%$ & $3.3 \%$ & $23.0 \%$ & $32.1 \%$ & $23.8 \%$ & $31.4 \%$ \\
\hline & PA & $-23.1 \%$ & $-12.5 \%$ & $-24.1 \%$ & $-11.5 \%$ & $-21.8 \%$ & $-10.7 \%$ & $-20.9 \%$ & $-11.6 \%$ \\
\hline & RO & $-7.0 \%$ & $-0.7 \%$ & $-7.6 \%$ & $-0.1 \%$ & $8.0 \%$ & $22.4 \%$ & $9.1 \%$ & $21.2 \%$ \\
\hline & $\mathrm{RR}$ & $-2.6 \%$ & $9.4 \%$ & $-3.8 \%$ & $10.6 \%$ & $14.1 \%$ & $34.1 \%$ & $15.7 \%$ & $32.5 \%$ \\
\hline \multirow{3}{*}{ ๖े } & PR & $-0.4 \%$ & $6.0 \%$ & $-1.0 \%$ & $6.6 \%$ & $0.0 \%$ & $6.2 \%$ & $0.5 \%$ & $5.7 \%$ \\
\hline & RS & $0.0 \%$ & $0.0 \%$ & $0.0 \%$ & $0.0 \%$ & $0.0 \%$ & $0.0 \%$ & $0.0 \%$ & $0.0 \%$ \\
\hline & SC & $4.4 \%$ & $10.6 \%$ & $3.9 \%$ & $11.1 \%$ & $-1.9 \%$ & $12.8 \%$ & $-0.7 \%$ & $11.6 \%$ \\
\hline \multirow{4}{*}{$\begin{array}{l}\frac{w}{5} \\
\text { 岁 } \\
\stackrel{\rho}{\omega}\end{array}$} & ES & $-13.0 \%$ & $-7.7 \%$ & $-13.5 \%$ & $-7.2 \%$ & $1.5 \%$ & $19.9 \%$ & $3.0 \%$ & $18.5 \%$ \\
\hline & MG & $-12.6 \%$ & $-2.7 \%$ & $-13.6 \%$ & $-1.7 \%$ & $-4.0 \%$ & $19.3 \%$ & $-2.1 \%$ & $17.4 \%$ \\
\hline & RJ & $-8.5 \%$ & $45.9 \%$ & $-13.7 \%$ & $51.1 \%$ & $-12.6 \%$ & $44.3 \%$ & $-8.0 \%$ & $39.7 \%$ \\
\hline & SP & $14.8 \%$ & $73.2 \%$ & $9.2 \%$ & $78.8 \%$ & $9.2 \%$ & $59.4 \%$ & $13.3 \%$ & $55.4 \%$ \\
\hline
\end{tabular}

